\title{
Conduct Clinical Trials
}

National Cancer Institute

\section{Source}

National Cancer Institute. Conduct Clinical Trials. NCI Thesaurus. Code C15793.

Conduct clinical trials and develop new trial methodologies. Trials should be designed in order to rapidly develop new therapeutic strategies, optimize clinical efficacy and proper use of available modalities, define factors that affect the success of therapeutic strategies (including the role of resistance), and advance our understanding of disease pathogenesis and progression. 